\title{
Towards an Integrated View of Literacy
}

Writing has been invented many times but the alphabet only once, according to Walter Ong in his book Orality and Literacy. His point is that there are many different ways of representing the world, which form the basis of the earliest writing systems. What is radically different about alphabetic scripts is that they do not represent objects in the world, but spoken language. The alphabetic principle means that written characters represent speech sounds. Those characters resemble nothing but themselves and have an entirely arbitrary relationship with the sounds they represent. Hence an important part of the history of writing is the establishment of conventions identifying the sounds that are relevant to be distinguished in the stream of speech and determining the representations to be used for those sounds.

In fact, writing technologises the spoken word. The technologies used have evolved over the history of writing, with the invention of printing and the Internet as key milestones, but that first invention remains as fascinating as ever-not least when new people are introduced to the world of writing. Reading in the sense of mastering the technology of writing is about entering the script in order to be able to enter texts and the world of meaning that is hidden inside them. However, it is also important to step outside the text to view it from a position of independence and mastery, which amounts to entering a community of written culture.

Such an historical and progressional approach to literacy actually has the potential to bring the research field together, in that this field can then be seen as dealing with a single issue of access, but at three different levels. These levels are most often treated separately in contemporary literacy research. The first level deals with access to writing and not least with difficulties gaining access to it. This is a large and well-established field using a wide range of methods, from case studies to experimental designs in controlled settings. The second level concerns access to texts and is investigated by reading and writing researchers who take an interest in individuals' cognitive processes and sometimes also in how the environment affects individuals' skills and performance. These two traditions share the same favourite hunting ground: the classroom. The third level, access to textual cultures, represents a unifying concept in the research field often referred to as new literacy studies (NLS), which is well established in Anglo-American countries. The founding idea of NLS was to break away from the belief that literacy and school are two sides of the same coin. This led to a move away from the individual perspective and from educational contexts, and to an active search for meaningful patterns in social 


\section{Atle Skaftun, Oddny fudith Solheim and Per Henning Uppstad}

situations outside school where writing and texts are used. Each of these three traditions has its focal points and its blind spots; together they should be able to provide a much richer picture of what literacy is than each can do on its own. We hope that the $N \mathcal{F L R}$ will be an appropriate forum for fruitful interaction between the three traditions.

We keep hearing about how we are living in a society marked by text-intensive communication, with little concrete descriptions and analyses to underpin such abstract characterisations. We need pictures of literacy practices in various aspects of life in the Nordic countries to understand our time and also to understand ourselves. Knowing more about present-day literacy practices will also equip us to study the past. Together, such pictures of present and past literacy practices will place us on a solid pedestal towards making wise choices in our endeavour to further develop the Nordic societies.

Schools have an urgent need to know what characterises the literacy practices of the various subjects they teach to turn the ideals of the school of the future into reality - to create a school where pupils are invited to participate as problem solvers and as producers of meaning. Practices define frameworks and roles, which may be flexible or rigid, and they represent values and ideologies. The pupils' individual development takes place within such frameworks. When it comes to literacy issues, early intervention is the order of the day. Strictly speaking, this can be construed as a principle calling for quick responses to the differing needs of different pupils- from mastering the written code to gathering the requisite textual experience for in-depth understanding and critical judgement. Furthermore, the issue of what pupils learn and how they develop is inextricably bound up with that of how they should be assessed and evaluated, which is probably one of the hardest points agree upon. The aim of obtaining a full picture of the development of children and young people within the framework of the literacy practices of the educational system represents a large and ambitious project to which the $N F L R$ will strive to contribute.

We also need studies shedding light upon and discussing key concepts; practice, depth, judgement, and development are all examples of vague concepts that call for interpretation and explicit reflection. Moreover, once we start engaging in conceptual clarification, discussions pertaining to the philosophy of science and to theory and methodology are only a short step away. Discussions and clarifications of this kind are important and necessary to unify and consolidate Nordic literacy research into a single - albeit complex - field.

The $N F L R$ wishes to be a forum for the production of knowledge and scholarly reflection about literacy in people's lives - in and out of school - today, in the past, and in the potential future. It invites scholars from traditions of reading and writing research that are established in the Nordic countries as well as scholars who position themselves closer to NLS to publish their work. In addition, it would be desirable for the $N f L R$ to conduct literacy research across the divide between the educational sector and the rest of society. Literacy is part of everybody's life, from beginning to end, and in almost all facets of society. The $N \mathscr{f L R}$ will contribute a broader and deeper understanding of written language practices and of the itinerary from 
the very first encounter with writing, over the almost fantastic event of breaking the code and understanding what the text says, to masterful participation in a textual culture.

Atle Skaftun

Oddny Judith Solheim

Per Henning Uppstad 